\title{
Circuit Design of RF Remote Controller in Code Matching Mode
}

\author{
Weidong Zhang ${ }^{\mathrm{a}}$, Gengsheng Zheng ${ }^{\mathrm{b}}$, Qifu Liu ${ }^{\mathrm{c}}$ \\ ${ }^{a} X i$ 'an Communication Institute, Xi'an 710106, China \\ ${ }^{b, c}$ School of Computer Science \& Engineering, Wuhan Institute of Technology, Wuhan 430073, China
}

\begin{abstract}
The concept and the state of the art of RF are reviewed firstly, as well as the superiority over the infrared. Then the design of the software and circuits of RF remote controller in code matching mode (RFRCCMM) is introduced, as well as the chip selection and the interface of SPI. The design approach of RFRCCMM is presented with the interpretation about the distribution of components and the layout.
\end{abstract}

Index Terms: Component; RF; SPI; PCB;

(C) 2011 Published by MECS Publisher. Selection and/or peer review under responsibility of the International Conference on E-Business System and Education Technology

\section{Introduction}

The TV remote control (RC) has transmitted signals with the infrared for a long time. However, with the development of RF, more and more signal interfaces of RC are changing from the infrared to RF. As a kind of high frequency electromagnetic wave with the frequency from $300 \mathrm{KHz}$ to $30 \mathrm{GHz}$, RF has the ability of long distance transmission, which results in more energy saving, more working life, and the miniaturization of RC. $\mathrm{RF}$ is widely applied in RC devices, such as wireless mouse and keyboard, and TV RC. RC is often required to be pointed to the receiver in the traditional TV RC operation based on the infrared. However, the complex environment of modern living room and the change of TV such as size make RC difficult to operate well. Because the transmission of RF signal is not restricted in straight-line propagation, RF RC can work well. Although RF RC is non-conditional, the high cost of its components and materials prevents it from popularity. Then the low cost design of RF RC is an important and hot issue.

The state of the art of RF and its superiority is argued in this paper. The design of the software and circuits of RFRCCMM is introduced, as well as the chip selection and the interface of SPI. Especially, the design approach of RFRCCMM is described deeply, which is on the basis of the platform of Protel 99 SE ( $[1]$, $[2],[3]$ ).

\footnotetext{
* Corresponding author.

E-mail ${ }^{a}$ zwdit@sohu.com, ${ }^{b}$ zhenggengsheng@sina.com
} 


\section{Overall Design of RF Remote Controller}

The RFRCCMM is an important branch of RF RC, so its design, arts and crafts are similar to traditional RF RC. In this section, the design of the software and circuits of RFRCCMM is introduced briefly.

\section{A. Overall Design of Software}

The actual steps are as follows.

Before data transfer, the transmitter and the receiver must match code, namely, they must adjust their frequency channels to two neighboring channels.

In the sleep mode, the interface such as P0 may sleep. When there is a change in the level of P0, the interface is awakened and the system enters into the polling mode for key press. When the event of key press is dealt with, the system reenters into sleep mode with a breakpoint. A new awakening operation will begin from this breakpoint. In sleep mode, the system has only 30ua while there are 30ma in normal mode.

The corresponding codes are as follows.

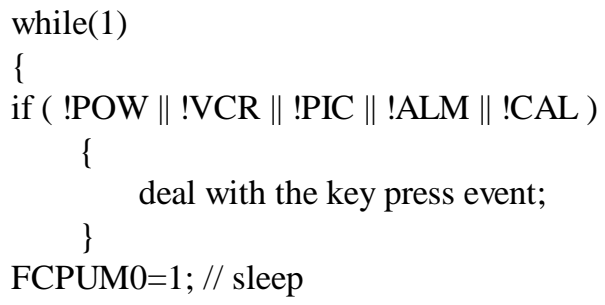

\section{B. Overall Design of Hardware}

The circuit of RFRCCMM consists of RF receiver, processing unit, power supply and I/O devices. The components will be introduced later.

According to the system architecture, the circuit diagram of RFRCCMM drawn with Protel 99 SE is shown in Fig.1.

TABLE I CHIP SELECTION OF RF REMOTE CONTROLLER

\begin{tabular}{|c|c|l|l|}
\hline Module & Function & IC Type & \multicolumn{1}{|c|}{ Feature } \\
\hline MCU & $\begin{array}{c}\text { Processing } \\
\text { unit }\end{array}$ & SN8P2612 & $\begin{array}{l}\text { OTP ROM size: } \\
\text { 2K } * 16 \text { bits; } \\
\text { RAM size: } \\
64 * 8 \text { bits; } \\
\text { 4-levels stack buffer }\end{array}$ \\
\hline TRANSCEIVER & $\begin{array}{c}\text { RF } \\
\text { Transceiver }\end{array}$ & M05D-C & $\begin{array}{l}\text { Wireless services in } \\
\text { 2.4GHz ISM band }\end{array}$ \\
\hline POWER & $\begin{array}{c}\text { Power } \\
\text { Supply }\end{array}$ & LP4054 & USB charge mode \\
\hline I/O & Input/Output & $\begin{array}{l}\text { 5-Button } \\
1-\text { Lamp }\end{array}$ & Diode signal output \\
\hline
\end{tabular}




\section{Detailed Design in Circuit}

\section{A.. Design of Chip Selection}

For the design of the circuits of RFRCCMM, the important components are listed in Table.1. We will introduce them briefly. SN8P2612 SCM: SN8P2612 SCM operates on the RISC-like platform and consists of $2 \mathrm{~K}^{*} 16$ bits program memory (OTP ROM), 64*8bits data memory (RAM), four interrupts (T0, TC0, INT0, INT1), two 8bits timer counters (TO, TCO), one-channel PWN output (PWM0), one-channel BUZZER output (BZ0) and four-classes stack buffer.

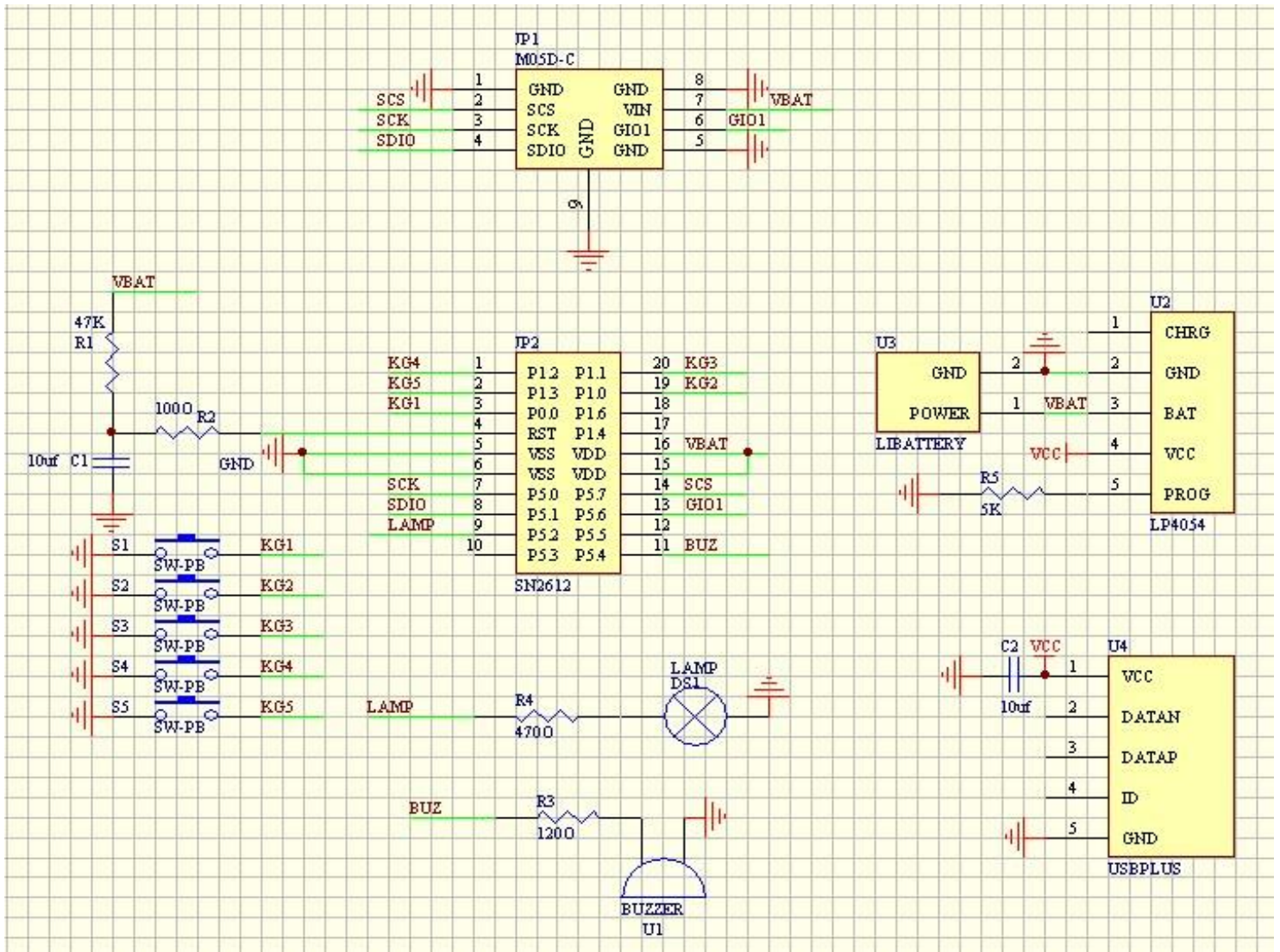

Fig. 1 Schematic diagram of RF RC

M05D-C: M05D-C is an IC with 500kps data speed for 2.4GHz ISM wireless services. Its RF module has 160 frequency channels and 16 different channels are selected here. The frequency hop (channel handoff) is carried out in every $20 \mathrm{~ms}$. In order to alleviate RF interference, the frequency of RF channels in transceiver changes in every $20 \mathrm{~ms}$.

LP4054 charger: The RFRCCMM is charged with USB interface, which is based on LP4054 charger chip. LP4054 is a constant-voltage/constant-current power supply, and can charge single lithium battery. It is very fit for portable USB devices. 
A7105 [4] RF chip: A7105 is a low cost RF IC for 2.4GHz ISM wireless services. Instead of the RC, it is used in the receiver side of $\mathrm{RC}$ signal. With an inner high sensitive receiver, it can reduce the RF emission greatly in the applications near 10m, which avoids the possible harm to human body.

\section{B. Design of SPI Communication}

SPI (Serial Peripheral Interface) bus is a synchronized serial device interface standard by Motorola, which is used in the full-duplex, synchronized serial communication between CPU and external devices. Because it has powerful hardware capability, its corresponding SPI software is so simple that CPU has more time to deal with other things.

SPI can transmit and receive serial data simultaneously. In most cases only four I/O SPI lines are needed for the communication between CPU and external devices. They are serial clock line (SCK), Main device Input/Secondary device output line (MISO), Main device output/Secondary device input line (MOSI) and Low Level effective Secondary device selections line (SS).

The SPI interface of A7105 may be 3 lines or 4 lines, here we use 3 lines interface. They are SCS, SCK and SDIO respectively. SCS is used in the control of SPI enabler. SCK controls the synchronization clock. SIDO is used in data $\mathrm{I} / \mathrm{O}$.

The interface connection between MCU and A7105 is shown in Fig.2.

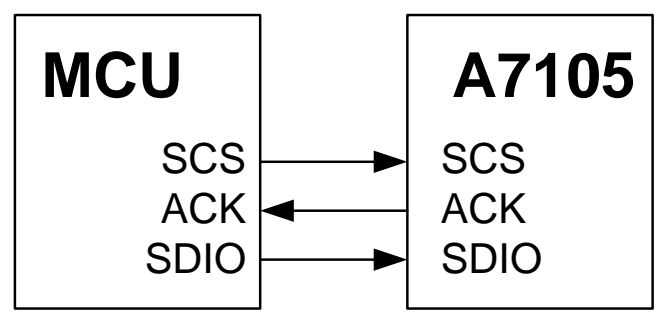

Fig.2 SPI communication between MCU and A7105.

The R/W operation of A7105: When a rising edge of SCK comes, the information is written in the control register. Having written the address information and a rising edge comes, MCU can read information (RF chip will transform the information when a falling edge comes.). The working cycle of SPI is shown in Fig.3 (writing operation) and Fig.4 (reading operation).

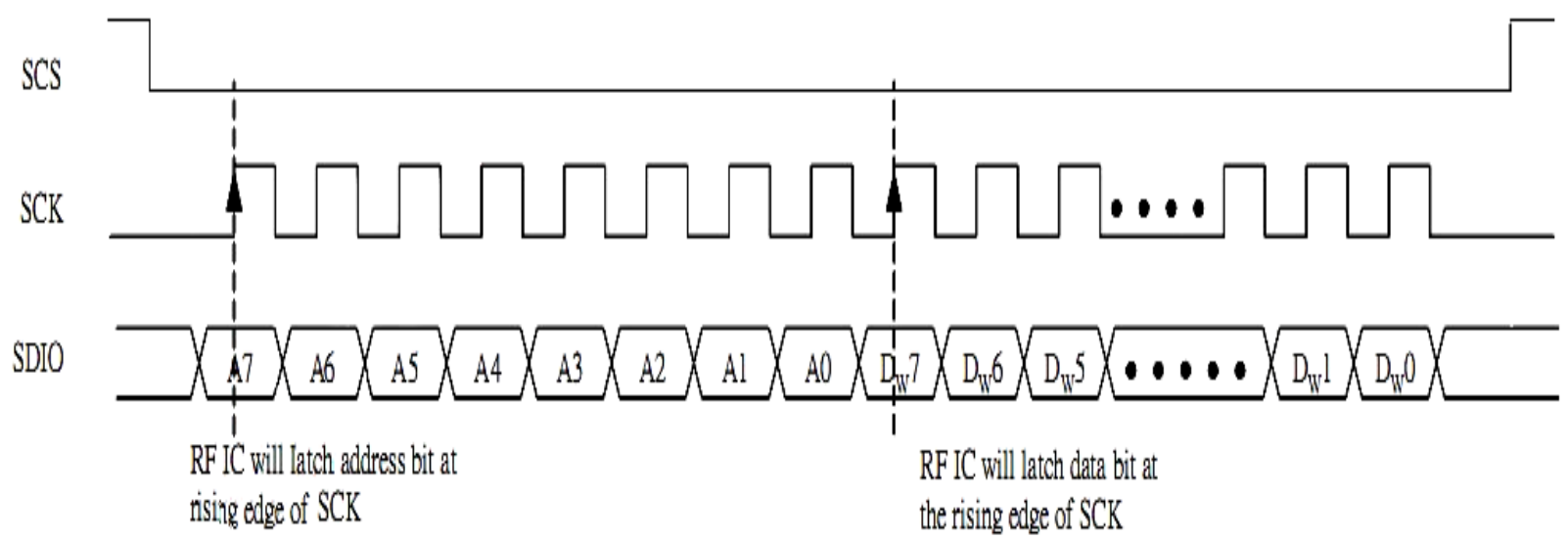

Fig.3 SPI timing simulation of writing operation 


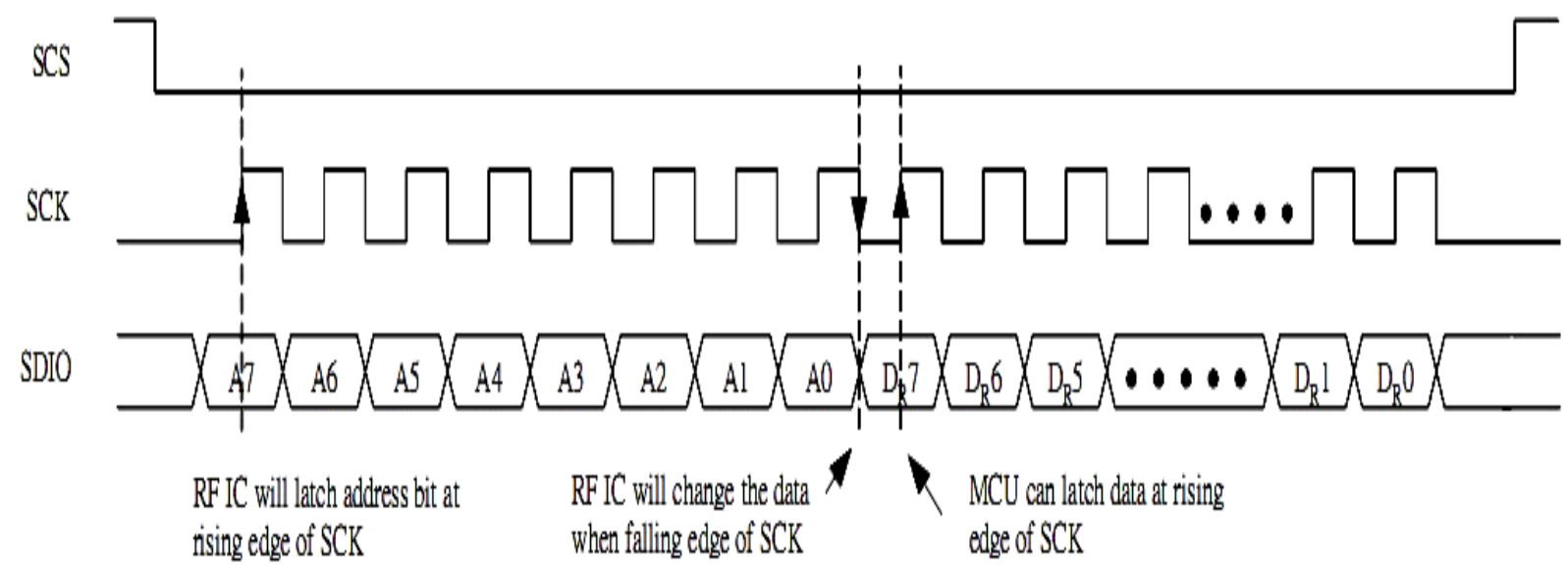

Fig.4 SPI timing simulation of reading operation

\section{Design of $P C B$}

The PCB consists of two layers, the top layer and the bottom layer. Both of them are metallized with copper, and the middle part is insulated. In this step, the layout and the distribution of components are the main design task.

The rules of the layout of components are as follows:

The circuits for the same function should be laid as near as possible, and the components should be adjusted so that the tracks are simple, which is also compatible to the layout of modules.

Pay more attention to the installation position and pressure of the components with big weight. The heat emission components should be mounted far from hot sensitive ones, even taking hot convection into consideration.

The drivers of I/O should be laid as near the edge of PCB and the plug extension as possible.

The density of layout should be even. The size (such as height and area) of components and the relative position should be considered for the electric performance and the producing and mounting feasibility.

The layout after adjustment is shown in Fig.5.

The approaches of line layout consist of automatic, semi-automatic, and manual. The most often approach is the mixture of automatic layout and manual layout. The layout rules such as safe distance, the degree of broken line and topology rule should be set at the beginning. After the layout, the manual adjustment is needed to wider the power lines and the ground lines, and increasing the interfaces of signal input and output.

The rules of line layout are as follows:

In most of cases, the power and ground lines should be laid firstly for the electric performance of PCB. The power and ground lines should be wider as far as possible. From the widest to the end, they are ground line, power line and signal line respectively.

The special lines such as high frequency lines should avoid being parallel at the input and output in order to reduce reflection interference. The lines in near layers should be vertical enough each other to reduce parasitic coupling.

45 degree broken line is preferred to 90 degree broken line for the purpose of reducing the radiation of high frequency signal. 


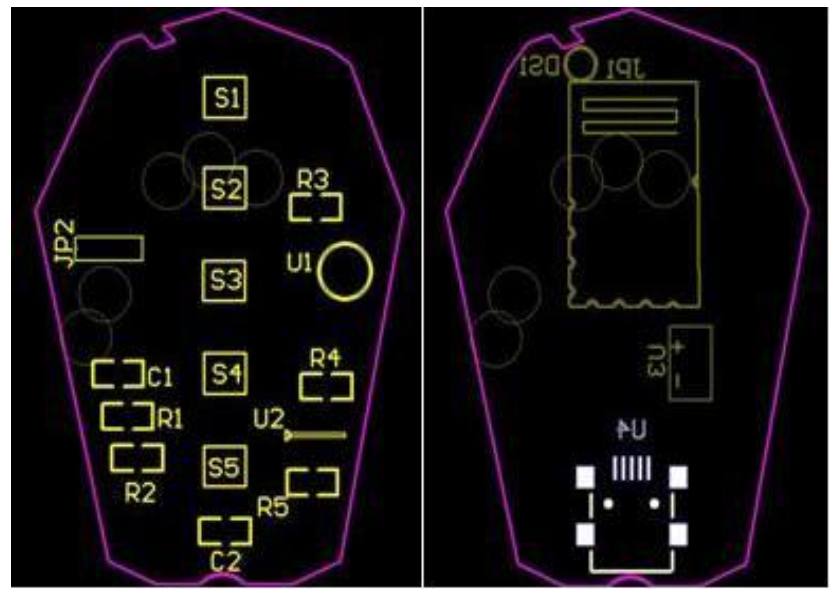

(1) Front end

(2) Back end

Fig.5 Layout design of PCB

The cycles should be avoided. If a cycle can not be avoided, it should be as small as possible. The lines via hole should be little.

After the principle layout, an optimization is needed. At the same time, the ground line with large area copper should be filled into the blank area if there is no error in DRC checkup. An alternative solution is to make multiple layers so that the power line and the ground line are not in the same layer.

The line layout of PCB is shown in Fig.6

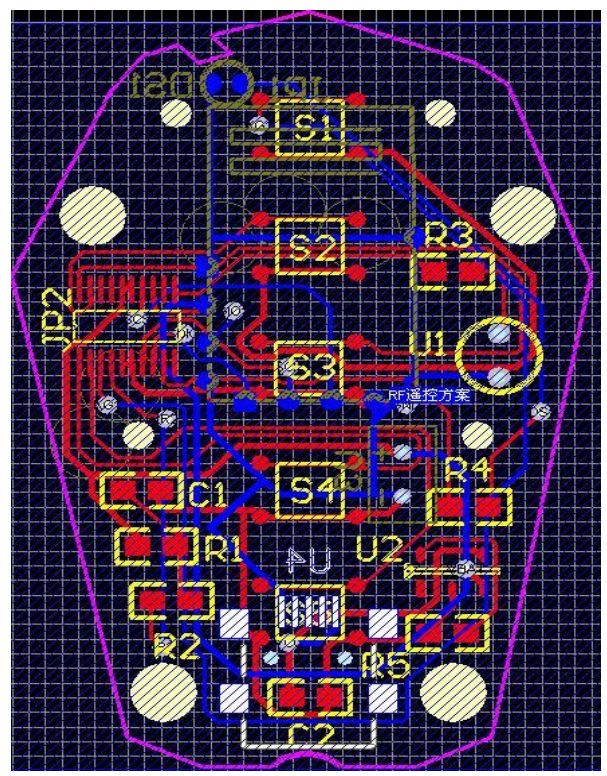

Fig.6 Wiring design of PCB 


\section{Conclusions}

$\mathrm{RF} \mathrm{RC}$ is a popular and developing technology for its performance superiority in recent years. That designing a low cost and compatible RF RC is very important. In this paper, the design of the software and circuits RFRCCMM is introduced with the design approach of RFRCCMM PCB, which has low cost and good performance.

\section{Acknowledgment}

It is a project supported by the Research Foundation of Education Bureau of Hubei Province, China (Grant No. Q20091501).

\section{References}

[1] J. Hu, "The elements of electronic CAD: Protel 99 SE," China electric power press, 2009.

[2] K. Liu, and Z. Gao, "Design examples and instructions of Protel 99 SE," Tsinghua university press, 2008.

[3] C. Huai, and Z. Li, "The elements and typical examples of circuits design with Protel 99 SE," Electronic industry press, 2008.

[4] AMICCOM, “A7105 2.4GHz Transceiver,” 2008. 